\title{
Performance evaluation and CFD multiphase modeling for Multistage Jatropha Fruit Shelling Machine
}

\begin{abstract}
The study involved performance evaluation of a Multistage Jatropha Fruit Shelling Machine (PI2015701163) which is used to produce Jatropha kernels (oil-rich nucleus) with higher purity by removing both the outer shells of fruits and husks (seeds' coating). The reduction of shells and husks together, which is not commonly practised at the moment, can improve oil yield and quality during oil extraction process in biodiesel production. The shelling machine consists of two cracking unit with each having different roller clearance for different stage of decortications. Every roller was featured with male threads on the surface. The male threads in different rotating directions and speeds can provoke more shearing effect onto the loaded Jatropha fruits. The machine design also deploys multistage separation mechanisms consisting of vibratory sieve and blowers for a purpose to produce cleaner kernels. The designed machine has been tested at different blower air speeds and fruit moisture contents. The optimal air speed and fruit moisture content were found to be $9.8 \pm 0.4 \mathrm{~m} \mathrm{~s}-1$ and around $5.7 \%$ w.b. respectively, where the husks could be removed without causing high kernel loss. The overall results show that the designed machine at the optimal conditions was able to remove $99 \%$ of shells and $52 \%$ of husks while controlling the kernel loss at around $5 \%$. Further, the separation process which applies blower as separation mean has been further analysed using Eulerian Model and Dense Discrete Phase Model (DDPM) in ANSYS Fluent, for a purpose to understand the behaviour of Jatropha fruits' particles under air flow during the separation process. The simulation revealed that some vortices have existed at converging portion or slope end of the designed separator duct due to instantaneous changes of air velocity and pressure. The formation of vortices could change the flowing direction of particles and lengthen the residence time of the particles in the duct. The phenomena were also found able to cause momentum loss of heavier kernel particles, achieving reduction of kernel loss during the separation process. The simulation results have provided a basis to understand the dynamics of multiphase flow. Further, the numerical model makes the study possible to provide a better insight for making a decision along with future developed scaling factor for machine scaled up activity.
\end{abstract}

Keyword: Jatropha fruit; Shelling machine; Kernel recovery; Multiphase model; CFD; DDPM 